\title{
Agôn
}

Revue des arts de la scène

7| 2015

La Distribution

\section{Une distribution nomade}

\author{
Ismaël Jude
}

\section{(2) OpenEdition \\ Journals}

\section{Édition électronique}

URL : http://journals.openedition.org/agon/3290

DOI : 10.4000/agon.3290

ISSN : 1961-8581

\section{Éditeur}

Association Agôn

\section{Référence électronique}

Ismaël Jude, «Une distribution nomade », Agôn [En ligne], 7 | 2015, mis en ligne le 05 octobre 2015, consulté le 19 avril 2019. URL : http://journals.openedition.org/agon/3290 ; DOI : 10.4000/agon.3290

Ce document a été généré automatiquement le 19 avril 2019.

Association Agôn et les auteurs des articles 


\title{
Une distribution nomade
}

\author{
Ismaël Jude
}

1 La distribution théâtrale suppose une sélection d'acteurs en vue d'une répartition des rôles; elle semble à première vue associée à la notion de partage. Or Gilles Deleuze dissocie, dans Différence et répétition, le concept de "distribution» qu'il qualifie de «nomade » de celui de « partage » qu'il nomme « sédentaire $»^{1}$. La notion de distribution intervient d'abord dans une problématique purement philosophique dont Deleuze n'envisage pas la portée théâtrale. «Un manifeste de moins ${ }^{2}$ » est un des rares textes où Deleuze se soucie d'aspects concrets du théâtre. Le nomadisme s'y trouve associé à la pratique de Carmelo Bene ${ }^{3}$.

2 Différence et répétition et «Un manifeste de moins» ont en commun d'articuler la conception "nomade " à une "critique de la représentation", la "représentation" désignant une illusion qui nous empêche de penser la différence en elle-même ${ }^{4}$. Deleuze énonce contre Brecht, dans "Un manifeste de moins ", une critique analogue à celle qu'il adresse à Kant, dans Différence et répétition. Brecht invente une méthode critique mais «ne pousse pas la "critique » assez loin ${ }^{5}$; Kant est accusé d'avoir "[trahi] la critique en même temps qu'il la concevait » ${ }^{6}$. Prisonniers de « la représentation », Kant ou Brecht en restent à un premier niveau de critique. La distribution nomade nous permet d'accéder à un deuxième niveau qui intègre une « critique de la représentation ». Une étude parallèle du clivage Brecht-Bene et de la distinction partage-distribution nous aidera à formuler l'idée d'une distribution théâtrale « nomade ».

\section{Racine grecque du nomade et du sédentaire}

Pour élaborer, dans Différence et répétition, la distinction entre partage et distribution, Deleuze a retenu les éléments fondamentaux de la thèse de doctorat d'Emmanuel Laroche ${ }^{7}$, une foisonnante étude philologique et linguistique de la racine et des dérivés de « nem » en grec ancien.

4 Laroche explique que, dans la langue homérique, le verbe "nemo", dont est issu notamment "nomade», n'a pas d'abord le sens de "je partage» mais celui de «je distribue $»^{8}$. La distribution désigne le geste qui consiste à donner, à offrir, et non pas 
celui qui vise à découper en plusieurs morceaux. Laroche prend l'exemple des dieux qui opèrent une distribution du destin aux hommes, et non un partage, « car la Fortune est infinie comme la toute-puissance divine elle-même... $»^{9}$ Ce qui est donné est d'abord indivis. Ainsi, le pâturage des nomades ne consiste pas en "terre découpée, lopin, morceau ${ }^{10}$ » que les troupeaux se partagent mais en un espace illimité où les animaux paissent. Le "lieu d'habitation sans limites précises " ${ }^{11}$ des nomades est un espace illimité et non pas la parcelle ou le district que bornent des frontières.

\section{Partage sédentaire de l'être chez Kant}

5 Deleuze suggère de concevoir l'être comme un tel territoire nomade: univoque, immanent, indivis, sans limites précises; les différences s'y distribuent «çà et là " $^{12}$. Il privilégie cette distribution nomade des différences dans l'être univoque au détriment d'un partage sédentaire de l'être ${ }^{13}$. Deleuze voit dans le partage sédentaire l'intervention de la «représentation ». Pour comprendre ce terme de "représentation », et la critique de Kant qu'elle vise, il faut avoir présent à l'esprit une distinction entre présentation et re-présentation.

Deleuze assimile le rapport entre l'expérience et la connaissance chez Kant au passage d'une présentation à une "représentation ». Le propre des phénomènes est qu'ils se présentent à notre sensibilité dans leur diversité. Le jugement synthétique rend possible la connaissance en appliquant les concepts de l'entendement à l'expérience: si nous pouvons connaître les phénomènes, c'est parce que notre subjectivité transcendantale applique à la diversité empirique les concepts ou catégories a priori (la causalité par exemple $)^{14}$. " À proprement parler, commente Deleuze, on ne dira pas que l'intuition même a priori soit une représentation, ni que la sensibilité soit une source de représentations. Ce qui compte dans la représentation, c'est le préfixe : re-présentation implique une reprise active de ce qui se présente (...). C'est la re-présentation elle-même qui se définit comme connaissance, c'est-à-dire comme la synthèse de ce qui se présente ${ }^{15}$. "

Deleuze désigne donc comme une illusion propre à la représentation le fait de calquer sur le découpage opéré par l'entendement un supposé partage de l'être en catégories ${ }^{16}$.

8 Les différences ne se partagent pas, n'apparaissent pas toutes faites comme des prédécoupages de l'être que nous n'aurions plus qu'à redécouvrir dans l'expérience; les différences se distribuent. Les différences entre les étants se produisent «çà et là » dans l'immanence de l'être. L'avantage d'une distribution nomade est donc de permettre de saisir le réel comme un milieu d'abord indivis, mais en devenir, où il s'agit de saisir les différenciations des étants dans leur processus même.

\section{Critique de la « tripartition hiérarchisée »}

9 Au théâtre, une distribution nomade se ferait sans répartition préalable des rôles. Une telle conception, proche de ce que Bruno Tackels désigne sous le nom d'écriture de plateau, marque la fin d'une hégémonie de l'auteur et d'une conception du metteur en scène comme "médiateur tout puissant entre le texte lu et la scène vue ${ }^{17}$, pour laisser place à un "geste initial des acteurs " ${ }^{18}$. Le nomadisme appelle un "théâtre des répétitions $»^{19}$; il évoque une distribution qui ne se produirait plus en amont mais au fil 
des répétitions. La répartition n'y est pas prédéterminée. La scène devient le point de départ de la création. C'est lors des répétitions que la partition se crée :

«Il s'agit en effet, écrit Tackels au sujet de Castellucci, de refuser la place hégémonique du texte écrit, et du même coup la pression tyrannique de la réalité du sens, ainsi que celle du sens de la réalité. Partant de ce principe iconoclaste, le théâtre construit une écriture qui part de la scène, une écriture dont l'auteur (les auteurs) s'origine(nt) dans la réalité du plateau, et se sert essentiellement des outils et de la grammaire qu'il met à sa portée. Au plus loin de la scène porte-voix d'un texte sacralement préexistant, cette posture iconoclaste puise, à même la scène comme à une source, tout ce qui va pouvoir s'y écrire. $»^{20}$.

10 Si Tackels reconnaît que le texte de Deleuze sur Bene "sonne aujourd'hui comme un programme du théâtre qui vient $»^{21}$, Deleuze, de son côté, ne désigne Bene ni comme un dramaturge ni comme un metteur en scène ni même comme un acteur mais comme un « homme de théâtre » ou un "opérateur ». Cette caractérisation peut être rapprochée de celle d' "écrivain scénique » : dans les deux cas, il s'agit d'expressions qui, parce qu'elles synthétisent les différentes fonctions théâtrales (auteur, metteur en scène, acteur), destituent leur « tripartition hiérarchisée ${ }^{22}$ » et les hégémonies qu'elles instaurent.

11 La conception antérieure à l'ère des "écrivains scéniques », nous la qualifierons de « hiérarchique »; elle repose sur un respect de la «tripartition hiérarchisée » de l'auteur, $\mathrm{du}$ metteur en scène et de l'acteur. Contemporaine de l'avènement de la figure du metteur en scène, elle connaît son apogée au vingtième siècle. L'histoire d'une défense du texte qui passe par l'intermédiaire de la mise en scène trouve ses racines chez André Antoine $^{23}$ aussi bien que dans le "théâtre d'art » russe de Stanislavski, elle se développe en France avec le Cartel (Jouvet, Dullin, Baty et Pitoëff) et avec l'idée d'un «théâtre populaire » de Firmin Gémier à Antoine Vitez.

12 Dans cette conception hiérarchisée, la distribution théâtrale présuppose, selon la féconde expression de Jean-Luc Nancy, le "partage des voix ${ }^{24}$ effectué par un auteur. Un metteur en scène attribue les répliques des personnages à tel acteur et telle actrice. « Distribuer » consiste à faire correspondre le nom d'un acteur au nom d'un personnage qui n'est lui-même qu'une répartition du texte. Un acteur ou une actrice, ayant pris connaissance de la partition qui lui a été attribuée, dispose du temps des répétitions pour s'efforcer de donner corps à ce partage effectué par l'auteur et repris tel quel par le metteur en scène. L'interprétation et l'ensemble des éléments scéniques sont mis en place en fonction de ce découpage inaugural du texte.

Bien sûr, chaque mise en scène, chaque interprétation d'un même texte sera différente. Chaque acteur s'emparera différemment de la partition qui lui aura été confiée. Seulement, cette différence n'intervient pas au commencement du processus des répétitions mais tout au bout d'une chaîne de partages sédentaires qu'elle ne fait que confirmer. Ce qui est sédentaire dans cette façon de pratiquer la distribution, c'est le jeu de correspondances mis en place à partir d'un partage originel. L'auteur, le metteur en scène et l'acteur mettent en jeu leur singularité mais tour à tour et dans un ordre hiérarchisé, au sein d'un partage du texte établi originellement par l'auteur du texte.

14 La distribution se fait à partir de "déterminations fixes", "assimilables à des "propriétés» ou des territoires limités dans la représentation » ${ }^{25}$. Les personnages apparaissent comme de telles déterminations. Jouer Hamlet ou interpréter Phèdre, c'est mettre en jeu sa différence mais au sein d'une répartition préalable du texte opéré par Shakespeare ou Racine. L'acteur qui se voit distribué le texte d'Hamlet n'est pas censé 
jouer en même temps certaines répliques d'Ophélie ou de Polonius. L'expression de la singularité de tel acteur et la singularisation du personnage se font à partir d'une délimitation préalable.

15 La distribution suppose une mise en relation entre le partage préétabli et l'interprétation. L'acteur ou l'actrice pourra correspondre plus ou moins à son rôle. Une démarche « nomade » tenterait de déstabiliser cette correspondance, introduisant un hiatus entre le rôle et l'acteur. La distribution la plus sédentaire en revanche serait celle qui chercherait à pousser la correspondance jusqu'à l'identification.

Force est de constater que cette conception sédentaire fonctionne plutôt comme un repoussoir dans une conception contemporaine du théâtre. Cette définition de la distribution s'apparente à un contre-modèle, à un modèle de ce qu'on ne veut plus faire. Une conception des personnages comme déterminations fixes est remise en cause aussi bien par les écritures modernes et contemporaines de Maeterlinck à Novarina, Koltès ou Sarah Kane que par les pratiques scéniques contemporaines de Carmelo Bene à Philippe Quesne en passant par Claude Régy ou Robert Wilson.

\section{Oppositions sédentaires...}

17 Dans quelle mesure Deleuze peut-il affirmer que Brecht reste prisonnier de la représentation ? Brecht a cherché à remettre en cause la fixité du personnage, à critiquer l'impératif d'une correspondance entre l'acteur et le rôle. Sa démarche est résolument critique par rapport à l'assimilation du personnage à une "détermination fixe». Brecht s'attaque à la rigidité du partage dans la distribution en intervenant, en amont déjà, sur le texte, et surtout, en aval, sur son interprétation. Il vise par là un certain aristotélisme aussi bien qu'un certain stanislavskisme. Il entend briser la belle unité du drame et le système de correspondances sédentaires : « les règles aristotéliciennes de la composition dramatique et les modes de jeu qu'elles impliquent ${ }^{26}$.

La pratique brechtienne est critique vis-à-vis de deux illusions principales: l'unité du drame et l'identification des acteurs aux personnages. Ces illusions empêchent les spectateurs de comprendre les rapports sociaux réels d'une époque déterminée. Brecht invente une écriture et un mode de jeu critiques qui ont pour but de faire apparaître cette réalité sociale. L'écriture épique et la mise en scène qui intègre le " Verfremdungseffekt " répondent à cette fonction. La démarche d'un metteur en scène "stanislavskien" consisterait à faire correspondre les rôles aux acteurs qu'il aurait choisis. La distribution s'apparenterait à une sélection, laquelle devrait se faire en vertu du partage préalable du texte. Cette sélection se ferait selon le critère d'une correspondance. Les acteurs sélectionnés auraient à faire correspondre leur jeu au rôle qui leur aurait été attribué. Brecht entend déstabiliser ce jeu de correspondances en introduisant des interruptions dans le drame, une étrangeté dans la mise en scène, une distance enfin entre l'acteur et le personnage. Brecht remet en cause la nécessité d'une correspondance entre le rôle et l'acteur. La relation de l'acteur à son rôle se fait au profit de "l'étrangeté » et au détriment des principes de la représentation telles que la ressemblance, l'identité ou la similarité.

Deleuze reproche cependant à Brecht de «ne pas sortir du domaine de la représentation » mais de « seulement passer d'un pôle dramatique de la représentation à un pôle épique de la représentation populaire ${ }^{27}$ ». Par «représentation », nous l'avons vu, Deleuze désigne 
une illusion qui nous conduit à soumettre la différence aux quatre figures de l'identité, de la ressemblance, de l'analogie et de l'opposition. Si Brecht demeure «prisonnier de la représentation » aux yeux de Deleuze, c'est parce qu'il conçoit la réalité sous la forme d'oppositions.

Ce que Brecht entend révéler, c'est un conflit latent de la société, une opposition entre les classes antagoniques, une contradiction qui doit trouver sa solution dans le dépassement dialectique que constitue la révolution prolétarienne. Brecht écrit par exemple deux scènes qu'il suggère d'insérer avant la fameuse « scène du balcon » de Roméo et Juliette ${ }^{28}$. On y voit Roméo, qui veut vendre un lopin de terre parce qu'il a besoin d'argent, congédier le vieux fermier qui y vivait avec toute sa famille. On y voit Juliette obliger sa servante à remuer une cuvette d'eau pour tromper un gardien qui monte la garde à sa porte, Juliette sachant pertinemment que sa servante manque un rendez-vous crucial avec son propre fiancé. Les deux prolétaires, qui rôdent pendant la scène d'amour au balcon, apparaissent comme la condition de possibilité matérielle de l'échange amoureux. Roméo et Juliette se trouvent ainsi dénoncés dans leurs conditions de vie bourgeoises. Ce qui est visé par Deleuze, c'est la dialectique ; ce n'est pas un hasard s'il procède avec une ironie similaire à celle qu'il emploie à l'encontre de Hegel.

21 "Sans doute y a-t-il la tentative de Brecht pour faire que les contradictions, les oppositions, soient autre chose que représentées ; mais Brecht lui-même veut seulement qu'elles soient "comprises", et que le spectateur ait les éléments d'une "solution" possible. $»^{29}$

Brecht entend nous faire prendre conscience du réel en interrompant les mécanismes de la représentation. Deleuze reconnait l'ambition du geste critique brechtien sans se satisfaire de sa conception du réel. La figure de l'opposition nous empêche d'apercevoir les différences telles qu'elles se produisent dans le réel. Il s'agit donc de "pousser la critique » au point de remettre en cause cette ultime illusion de la représentation.

«Mais pourquoi, demande Deleuze, les conflits sont-ils généralement subordonnés à la représentation, pourquoi le théâtre reste-t-il représentatif chaque fois qu'il prend pour objet les conflits, les contradictions, les oppositions? C'est parce que les conflits sont déjà normalisés, codifiés, institutionnalisés. Ce sont des "produits". Ils sont déjà une représentation, qui peut d'autant mieux être représentée sur la scène ${ }^{30}$.»

\section{... Différences nomades}

24 Ce que manque le théâtre épique, et ce que met en jeu le théâtre de Carmelo Bene, ce sont les petites différences en train d'advenir dans le réel. Il s'agit de saisir en deçà de la représentation, non plus des oppositions déjà produites mais la production du réel; il s'agit d'accompagner le mouvement même de la création, le nouveau, la différence en train de se faire $^{31}$, le devenir qui constitue le réel. Les pratiques de Brecht et Bene diffèrent du point de vue de leurs conceptions du réel. Ici, l'opposition structure le réel, là, il s'agit de saisir le réel comme différence. Dans son style lyrique inimitable, Deleuze précise :

"Quand un conflit n'est pas encore normalisé, c'est parce qu'il dépend d'autre chose de plus profond, c'est parce qu'il est comme l'éclair qui annonce autre chose et qui vient d'autre chose, émergence soudaine d'une variation créatrice, inattendue, sub-représentative. $»^{32}$ 
Deleuze octroie à cette profondeur sub-représentative une dimension éminemment politique qui prend une voie différente de la visée brechtienne. Il défend la politique minoritaire qui anime la pratique théâtrale de Carmelo Bene contre cette dialectique qui sous-tend la pratique brechtienne. L'analyse de Carmelo Bene lui fournit l'occasion d'affirmer la connexion entre défense du devenir et politique minoritaire qu'il a commencé à développer en collaborant avec Félix Guattari ${ }^{33}$ et qui se déploiera dans Mille plateaux $^{34}$ :

" On voit bien s'esquisser une politique à travers les déclarations ou les positions de $\mathrm{C}[$ armelo] $\mathrm{B}[\mathrm{ene}]$. La frontière, c'est-à-dire la ligne de variation, ne passe pas entre les maîtres et les esclaves, ni entre les riches et les pauvres. Car, des uns aux autres, se tisse tout un régime de relations et d'oppositions qui font du maitre un esclave riche, de l'esclave un maître pauvre, au sein d'un même système majoritaire. $\rrbracket^{35}$

Les deux pratiques divergent non seulement dans leurs conceptions du réel mais encore dans leurs définitions de ce que sont un peuple et un individu. Deleuze note que Carmelo Bene ne croit pas « à un théâtre populaire, à un théâtre pour tous, à une communication de l'homme de théâtre et du peuple $\aleph^{36}$. C'est moins une aventure thêâtrale définie historiquement que Deleuze désigne à travers le «théâtre populaire » qu'une conception $\mathrm{du}$ « peuple $»^{37}$ :

"C'est que, quand on parle d'un théâtre populaire, on tend toujours vers une certaine représentation des conflits, conflits de l'individu et de la société, de la vie et de l'histoire, contradictions et oppositions de toutes sortes qui traversent une société mais aussi les individus. $\|^{38}$

Deleuze propose que le peuple et l'individu ne soient plus envisagés comme des institutions déjà partagées mais comme des processus que parcourt le devenir. Il reprend cette formule de Paul Klee qui dit que « le peuple manque »:

«Il faut que l'art, particulièrement l'art cinématographique, participe à cette tâche: non pas s'adresser à un peuple supposé, déjà là, mais contribuer à l'invention d'un peuple. $\rrbracket^{39}$

Le peuple est à venir ; il doit être inventé par les minorités. Il ne faut pas rabattre des divisions extérieures sur une idée préconçue, essentialiste, du peuple. Il faut se rendre sensible aux devenirs révolutionnaires qui vont conduire un peuple à se soulever, qui vont amener une entité d'abord indifférenciée, inexistante, n'existant "pas encore », à devenir un peuple en se soulevant. Ni le peuple ni l'individu ne doivent être conçus comme des déterminations fixes et préalables. Peuple et individu se trouvent pris dans le même devenir-minoritaire, «car, si le peuple manque, s'il éclate en minorités, c'est moi qui suis d'abord un peuple, le peuple de mes atomes, comme disait Carmelo Bene $»^{40}$. Peuple et individu doivent être envisagés comme des masses indéterminées, ressemblant au territoire nomade indivis. Les devenirs mineurs vont produire des différences au sein de cette masse d'abord indéfinie. Le partage sédentaire considère l'individu comme une détermination fixe. La distribution nomade, en revanche, met en jeu les devenirs au sein de l'individu.

Le travail critique qu'effectue Carmelo Bene sur les pièces de Shakespeare (Roméo et Juliette et Richard III) remet en cause la tripartition hiérarchisée en se déployant dans la réécriture, la mise en scène et l'interprétation. Deleuze est très intéressé par le fait que Carmelo Bene ne procède alors pas par ajout (comme c'est le cas de Brecht), mais par soustraction. "L'amputation» du texte est comparée par Deleuze à une opération chirurgicale qui va permettre à des développements virtuels d'avoir lieu : 
«Alors toute la pièce, parce qu'il y manque maintenant un morceau choisi non arbitrairement, va peut-être basculer, tourner sur soi, se poser sur un autre côté. Si vous amputez Roméo, vous allez assister à un étonnant développement, le développement de Mercuzio, qui n'était qu'une virtualité dans la pièce de Shakespeare. $»^{41}$

Bene choisit de supprimer un élément autour duquel se cristallise la représentation. Il ne recompose pas le sens à partir d'éléments qui s'ajoutent dans une interruption, comme le fait Brecht avec la prise en compte de la réalité sociale ; il trouble la représentation à partir d'éléments qu'il soustrait. Comme on scie la poutre maîtresse d'un édifice, il fait s'effondrer le partage préalablement établi du sens. Deleuze insiste sur le fait que le choix de Carmelo Bene ne porte pas sur n'importe quel élément mais sur des éléments « majeurs » de la pièce. Cette amputation des éléments majeurs rend possible le développement d'éléments mineurs.

31 Le premier geste de réécriture critique concernant Richard III consiste à supprimer tous les rôles masculins à l'exception de Gloucester lui-même (le futur Richard III) de sorte que l'intrigue de la pièce va tourner autour de la relation de Richard III aux femmes :

«Mais alors apparaît sous une nouvelle lumière ce qui n'existait que virtuellement dans la tragédie. Richard III est peut-être la seule tragédie de Shakespeare où les femmes ont pour leur compte des rapports de guerre. Et Richard III, de son côté, convoite moins le pouvoir qu'il ne veut réintroduire ou réinventer une machine de guerre, quitte à détruire l'équilibre apparent ou la paix de l'Etat [...]. En opérant la soustraction des personnages de Pouvoir d'Etat, C[armelo] B[ene] va donc donner libre cours à la constitution de l'homme de guerre sur scène, avec ses prothèses, ses difformités, ses excroissances, ses malfaçons, ses variations. ${ }^{42}$

Deleuze distingue dans Différence et répétition deux types de « sélection », l'une, sédentaire, « revient au même » ou « fait revenir le même » mais l'autre, nomade, fait « revenir la différence ». Voici comment Carmelo Bene opère une telle « sélection de la différence »: des éléments isolés se développent contre la totalité. Les éléments virtuels se développent contre les éléments de Pouvoir qui soutiennent la représentation habituelle de Richard III, le nomade contre le sédentaire, le virtuel contre le réel, le devenir contre la représentation, le mineur contre le majeur, les machines nomades contre l'appareil d'État sédentaire $^{43}$, l'homme de guerre contre les hommes d'État, les femmes contre les hommes.

On reprocherait à tort à Deleuze de réintroduire de la sorte des oppositions. "Se développer contre " ne désigne pas une opposition mais une "excroissance», un développement inattendu de la différence qui mine la représentation de l'intérieur. De même, le virtuel ne s'oppose pas au réel. Le virtuel est une dimension du réel qui peut connaître un développement propre. Les développements virtuels produisent une différenciation interne du réel ${ }^{44}$. La machine de guerre nomade ne s'oppose pas à l'appareil d'État sédentaire. Gloucester appartient à la «lignée » des chefs d'État, il s'y " glisse » comme « un personnage inquiétant », monstrueux, qui « annonce dès le début son intention de réinventer une machine de guerre et d'en imposer la ligne (difforme, fourbe et traître, il se réclame d'un «but secret » tout autre que la conquête du pouvoir d'Etat, et d'un autre rapport avec les femmes). ${ }^{45}$ De même, lorsqu'on parle de " l'Homme » comme espèce, les femmes appartiennent bel et bien à cette représentation majoritaire mais elles y appartiennent comme une part manquante ou comme un peuple qui n'existe pas encore. Un développement virtuel de cette dimension féminine peut déstabiliser la représentation majoritaire de l'espèce humaine. 

ressortir les devenirs qui constituent un personnage. On cesse de considérer le personnage comme un individu déjà constitué pour mettre en jeu la constitution du personnage. On ne s'intéresse pas au commencement et à la fin, à la naissance et à la mort du personnage, mais au processus de son individuation, à son devenir. En ce sens, la distribution nomade « sélectionne la différence » ou « fait revenir la différence ». Il n'est plus demandé aux acteurs de correspondre ou de ressembler à leurs personnages mais de les entraîner dans des différenciations virtuelles ou des devenirs-mineurs. C'est en vertu de cette mise en jeu des différences que le théâtre de Carmelo Bene ouvre une voie nouvelle.

Ni l'actrice qui joue Lady Anne ni Bene qui joue Gloucester ne font correspondre leur jeu à une Lady Anne ou à un Gloucester déterminés dans le cadre d'une représentation préconçue de la pièce, ils produisent la «mise en variation» d'une multiplicité de Gloucester et de Lady Anne virtuels qui constituent autant de développements mineurs de Gloucester ou de Lady Anne :

«Supposons que Lady Anne dise à Richard III : “Tu me fais horreur !" Ce n'est à aucun égard le même énoncé suivant qu'il est le cri d'une femme de guerre, celui d'un enfant devant un crapaud, celui d'une jeune fille qui éprouve une pitié déjà consentante et amoureuse... il faudra que Lady Anne passe par toutes ces variables, qu'elle se dresse en femme de guerre, régresse en petit enfant, renaisse en jeune fille, sur une ligne de variation continue, et le plus vite possible. $»^{46}$

Il ne suffit pas que l'acteur ne corresponde pas à son rôle, qu'il soit sélectionné en dehors de principes dominants de la représentation: la ressemblance, la vraisemblance, la similarité ou l'identité. Le contre-emploi ne fait pas une distribution nomade. Même un déplacement de la distribution peut confirmer le partage sédentaire des sexes, des âges et des classes sociales. Il y a une façon parfaitement sédentaire pour un homme de se travestir en femme ou pour une vieille actrice de jouer le rôle d'une jeune fille. De Marivaux à Feydeau, une mise en scène des différences sexuelles ou sociales peut parfaitement avoir la fonction de consolider l'ordre établi des dominations ${ }^{47}$. Les différences mises à l'épreuve aboutissent à un retour «à la normale ", à une paix sociale, une harmonie retrouvée ou une re-normalisation. Des différences y sont présentées mais, à la fin, tout revient au même. Les jeunes gens se marient, les hommes avec les femmes, les vieux retrouvent les vieux, les valets finissent ensemble, les maîtres ensemble, et ainsi de suite.

Une authentique distribution nomade suppose en revanche une métamorphose, un changement, une transformation non seulement du personnage mais, plus profondément, de l'acteur ou de l'actrice. La distribution ne pourra être considérée comme nomade qu'à condition que l'acteur ou l'actrice s'engage dans un devenir. Il ne s'agira pas de représenter une femme ou une jeune fille mais d'entrer dans un devenir-femme ou dans un devenir-jeune fille. Le jeu doit destituer les différences représentatives, le partage institutionnel en sexes, âges ou classes. L'écart brechtien entre l'acteur et son rôle est insuffisant. Il faut que l'acteur ou l'actrice mette en jeu une différence non seulement par rapport au personnage mais plus profondément par rapport à soi-même. La distribution nomade se traduit par une exploration des devenirs qui destitue et le personnage et l'acteur parce qu'elle destitue la conception sédentaire de l'individu. La distribution nomade doit donner lieu à une redéfinition du jeu d'acteur. Jouer consiste à mettre en variation les développements virtuels non seulement des personnages mais encore de l'acteur en tant que sujet.

Agôn, 7 | 2015 


\section{NOTES}

1. Gilles Deleuze, Différence et répétition, Paris, P.U.F., 1968, p. 53-57.

2. Gilles Deleuze, "Un manifeste de moins », in Carmelo Bene et Gilles Deleuze, Superpositions, trad. Jean-Paul Manganaro et Danielle Dubroca, Paris, France, Éd. de Minuit, 1979.

3. Le concept de " nomade " a évolué et Deleuze ne l'associe plus guère au mot " distribution ».

4. Gilles Deleuze, Différence et répétition, op. cit., p. 44-45 et p. 341-346. Voir Ismaël Jude, Gilles Deleuze, théâtre et philosophie : la méthode de dramatisation, Mons, Belgique, Éd. Sils Maria, 2013, p. 73-79.

5. Gilles Deleuze, « Un manifeste de moins », op. cit., p. 121.

6. Gilles Deleuze, Nietzsche et la philosophie, Paris, P.U.F. [1962], « Quadridge », 1997, p. 59.

7. Emmanuel Laroche, Histoire de la racine nem - en grec ancien, Paris, Klincksieck, 1949.

8. Ibid., p. 7-8.

9. Ibid., p. 9.

10. Ibid., p. 116.

11. Ibid., p. 117.

12. Ibid., p. 124. Gilles Deleuze, Différence et Répétition, op. cit., p. 54.

13. Pour défendre l'idée que l'être est différence, Deleuze emprunte au philosophe médiéval Duns Scot la thèse de l'univocité de l'être. «L'univocité » répond au projet de comprendre comment la différence entre les étants se produit dans l'être. La majeure étant que : «l'être se dit en un seul et même sens ", la mineure qu'il se dit " de toutes ses différences individuantes », c'est-à-dire que " ce dont il se dit diffère », la conclusion est que l'être se dit de la différence elle-même. Deleuze fait jouer, dans sa critique de l'analogie kantienne, l'argument avec lequel Scot réfute Thomas d'Aquin. Gilles Deleuze, Différence et répétition, op. cit., p. 52-58, p. 386-389. Deleuze tire nombre de ses conclusions de l'ouvrage qu'Etienne Gilson consacre au philosophe médiéval : Jean Duns Scot, introduction à ses positions fondamentales, Paris, Vrin, 1952.

14. La liste exhaustive des douze catégories kantiennes est : unité, pluralité, totalité (quantité), réalité, négation, limitation (qualité), inhérence et subsistance, causalité et dépendance, communauté (relation), possibilité-impossibilité, existence-non-existence, nécessité-contingence (modalité). Emmanuel Kant, Critique de la raison pure, Paris, P.U.F [1944], « Quadridge », 1993, p. 94.

15. Gilles Deleuze, La philosophie critique de Kant, Paris, P.U.F. [1963], « Quadridge, 1988, p. 15.

16. Cette illusion est le fait d'une des quatre figures de la représentation : l'analogie - les trois autres figures étant l'identité, la ressemblance et enfin l'opposition. Deleuze récuse cette façon dont Kant fait correspondre, par analogie, les concepts transcendantaux à un découpage préalable de l'être.

17. Bruno Tackels, Écrivains de plateau. 1, Les Castellucci, Besançon, France, les Solitaires intempestifs, 2005, p. 13.

18. Ibid., p. 19.

19. Deleuze désigne sous le nom de "répétition ", une opération, inspirée de l'éternel retour de Nietzsche et de la reprise de Kierkegaard. Cf Ismaël Jude, Gilles Deleuze, théâtre et philosophie : la méthode de dramatisation, op. cit., p. 124-219. C'est par cette opération sélective que la différence se produit. La répétition sélectionne la différence ou fait « revenir la différence ». À cet égard, le nom de «théâtre des répétitions » conviendrait parfaitement à l'idée d'un théâtre dont l'enjeu est la différence, une conception nomade de la pratique théâtrale, proche de ce que Tackels conçoit sous le nom d'écriture de plateau. Cette appellation de "théâtre des répétitions " 
présente le double avantage de désigner une affinité avec le concept deleuzien de répétition mais aussi de signifier que la différence se produit lors des répétitions. Notre attention au fait théâtral se déplace ainsi de l'auteur et du metteur en scène vers l'acteur, du drame et de la scène vers les répétitions.

20. Bruno Tackels, Écrivains de plateau. 1, op. cit., op. cit., p. 28. Nous soulignons : " porte-voix d'un texte sacralement préexistant ».

21. Ibid., p. 21.

22. Ibid., p. 28.

23. Cf André Antoine, Jean-Pierre Sarrazac et Philippe Marcerou, Antoine, l'invention de la mise en scène: anthologie des textes d'André Antoine, Arles, France, Actes Sud, 1999.

24. Jean-Luc Nancy, Le Partage des voix, Paris, Galilée, 1982.

25. Gilles Deleuze, Différence et répétition, op. cit., p. 54.

26. Bertolt Brecht, L'Achat du cuivre, éd. André Combes et Jean-Marie Valentin, Paris, France, l'Arche, 2008, p. 27.

27. Gilles Deleuze, «Un manifeste de moins », op. cit., p. 121.

28. Ibid., p. 158-161.

29. Ibid.

30. Ibid., p. 121-122.

31. Cf. Paola Marratti, «Le nouveau en train de se faire. Sur le bergsonisme de Deleuze ", in Revue internationale de philosophie, $\mathrm{n}^{\circ}$ 241, 2007/3, p. 261-271.

32. Gilles Deleuze, «Un manifeste de moins », op. cit., p. 122.

33. Voir notamment Gilles Deleuze et Félix Guattari, Kafka : pour une littérature mineure, Paris, France, Éd. de Minuit, 1975.

34. Gilles Deleuze et Félix Guattari, Mille plateaux, Paris, France, Ed. de Minuit, 1980, p. 434-623.

35. Gilles Deleuze, « Un manifeste de moins », op. cit., p. 126.

36. Ibid., p. 121.

37. N'ayant pas un goût démesuré pour le théâtre, Deleuze n'est pas doté d'une connaissance très approfondie de l'histoire du théâtre. Il assimile volontiers le théâtre épique et l'idée d'un "théâtre populaire " à la française, héritier d'un certain brechtisme. Si son intérêt pour Bene témoigne d'un réel engouement, sa divergence avec Brecht doit être envisagée d'un point de vue plus conceptuel.

38. Gilles Deleuze, «Un manifeste de moins », op. cit., p. 121.

39. Gilles Deleuze, L'Image-temps, Paris, Minuit, 1985, p. 283.

40. Ibid., p. 287.

41. Gilles Deleuze, « Un manifeste de moins », op. cit., p. 88.

42. Ibid., p. 90.

43. Sur le couple machine de guerre nomade et appareil d'Etat sédentaire, voir Gilles Deleuze et Félix Guattari, Mille plateaux, op. cit., p. 434-527.

44. Gilles Deleuze, Différence et répétition, op. cit., p. 269. Voir Ismaël Jude, Gilles Deleuze, théâtre et philosophie : la méthode de dramatisation, op.cit., p. 80-83.

45. Gilles Deleuze et Félix Guattari, Mille plateaux, op. cit., p. 438.

46. Gilles Deleuze, «Un manifeste de moins », op. cit., p. 104-105.

47. Cf. Ismaël Jude, Gilles Deleuze, théâtre et philosophie : la méthode de dramatisation, op. cit., p. 31. 


\section{RÉSUMÉS}

Gilles Deleuze fait jouer à Carmelo Bene vis-à-vis de Brecht un rôle comparable à celui que jouait, dans Différence et répétition, la « distribution nomade » par rapport au " partage sédentaire ». Une étude parallèle du clivage Brecht-Bene et de la distinction partage-distribution nous permet de formuler l'idée d'une distribution théâtrale « nomade ».

The role that Gilles Deleuze gives to Carmelo Bene towards Brecht is similar to that played by "nomadic distribution" towards "sedentary division" in Difference and Repetition. We are able to formulate the idea of a "nomad distribution" (in the sense of theatrical "casting") through a parallel study of the Brecht-Bene cleavage and the distinction between distribution and division.

\section{INDEX}

Keywords : Deleuze (Gilles), Bene (Carmelo), Difference and Repetition, Brecht (Bertolt), nomad, theater and philosophie

Mots-clés : Deleuze (Gilles), Bene (Carmelo), Brecht (Bertolt), Différence et répétition, nomade, théâtre et philosophie 